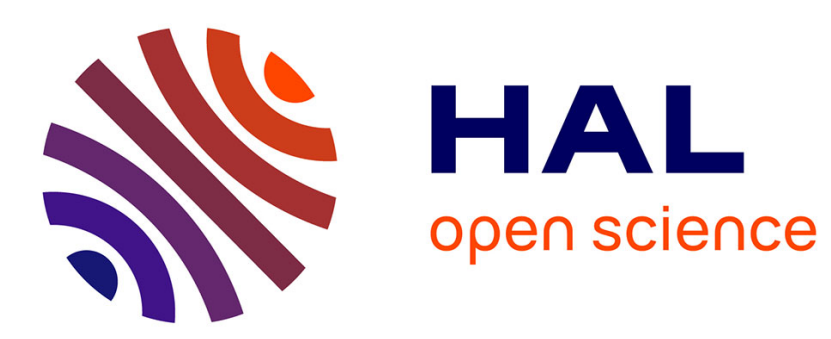

\title{
Variations of Solar Oblateness with the 22 year-magnetic Cycle Explain Measurements Apparently Inconsistent
}

\author{
Abdanour Irbah, Redouane Mecheri, Luc Damé, Djelloul Djafer
}

\section{To cite this version:}

Abdanour Irbah, Redouane Mecheri, Luc Damé, Djelloul Djafer. Variations of Solar Oblateness with the 22 year-magnetic Cycle Explain Measurements Apparently Inconsistent. The Astrophysical journal letters, 2019, 875 (2), pp.art. L26. 10.3847/2041-8213/ab16e2 . insu-02098484

\section{HAL Id: insu-02098484 \\ https://hal-insu.archives-ouvertes.fr/insu-02098484}

Submitted on 23 Apr 2019

HAL is a multi-disciplinary open access archive for the deposit and dissemination of scientific research documents, whether they are published or not. The documents may come from teaching and research institutions in France or abroad, or from public or private research centers.
L'archive ouverte pluridisciplinaire HAL, est destinée au dépôt et à la diffusion de documents scientifiques de niveau recherche, publiés ou non, émanant des établissements d'enseignement et de recherche français ou étrangers, des laboratoires publics ou privés. 
Draft Version April 18, 2019

Typeset using LATEX default style in AASTeX62

Variations of solar oblateness with the 22yr magnetic cycle explain measurements apparently inconsistent

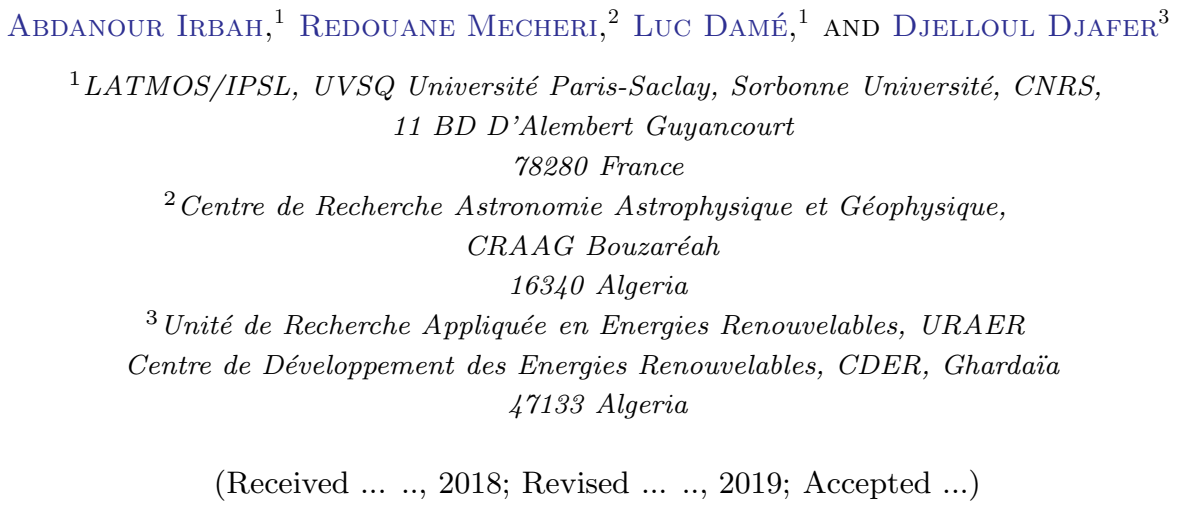

\section{INTRODUCTION}

The internal dynamics of the Sun from its depths to the most superficial layers is manifested by visible disturbances on the surface of the photosphere. They lead directly small deviations from the sphericity of the Sun. The solar shape thus reflects the internal state of the Sun and the processes that take place there. It soon drew the attention of Newcomb (1865), who asked why the Newtonian gravitational theory could not correctly predict the advance of Mercury perihelion observed by Le Verrier (1859). He suggested that a 500 milli-arcsecond (mas) oblateness i.e. the pole-equator radius difference, due to a rapidly rotating interior of the Sun could provide an explanation (Newcomb 1895). The solar shape involved two main issues at this stage, namely the theory of gravitation and the internal rotation of the Sun. The advent of Einstein's theory of General Relativity brought explanation to the anomalous advance of Mercury perihelion but it remains that good estimates of solar oblateness is of great importance for this theory i.e. gravitational moments of the Sun is still relevant for the Mercury perihelion (Chapman 2008). Many measurements of 
solar oblateness have been carried out for several decades, bringing other questions about its mean value and temporal variations. A good review on the history of oblateness measurements and the issues they have raised, may be found in the papers of Dicke \& Goldenberg (1974) and Rozelot \& Damiani (2011). Some scientific events need, however, to be recalled. The measurement of Dicke and Goldenberg's solar oblateness in 1966 in Princeton, New Jersey, is worth mentioning because of its high value $(41.9 \pm 3.3$ mas), which allowed them to highlight the scalar-tensor theory of gravitation and the quadrupole moment of the Sun associated to a rapidly rotating core (Dicke \& Goldenberg 1974). These results were widely criticized but have sparked growing interest in studying the interior of the Sun. Among the critics, Sturrok \& Gilvarry (1967) have shown that a rapid change of internal magnetic field would cause magnetic distortions at the surface resulting in an oblateness comparable to the observations. The magnetic field then appeared as the third major issue involved in the solar shape. Gravitational models with contribution of helioseismology, which probes Sun's interior to estimate both radial profiles of latitudinal differential rotation and the internal magnetic field (Goode \& Thompson 1992; Antia et al. 2000; Paterno et al. 1996), made it possible to identify acceptable values for oblateness mostly induced by the centrifugal force on surface layers with a very weak contribution from the gravitational quadrupole moment $J_{2}$ (Armstrong \& Kuhn 1999; Mecheri et al. 2004; Antia et al. 2008). Measurements made in space (Fivian et al. 2008; Irbah et al. 2014), on balloons (Egidi et al. 2006) and on ground (Hill \& Stebbins 1975; Rozelot et al. 2009) confirmed the expected values. Temporal variations are just as useful for understanding the functioning of the Sun in relation with its activity cycle. Particularly, helioseismic inversion revealed an insignificant temporal variation of $J_{2}$ with, in counterpart, variation of surface layers properties (Antia et al. 2008; Lefebvre et al. 2007) correlated with solar cycle, suggesting that shape variations of the Sun are certainly associated with the magnetic field. Therefore, accurate scrutiny of surface layers of the Sun is very important and requires high precision helioseismology for a good resolution of their structure (Reiter et al. 2015). Time series of oblateness recorded on ground, balloons and lately from space showed variations without, however, being conclusive. They are in phase (Damiani et al. 2011; Rozelot et al. 2009; Dicke et al. 1987; Emilio et al. 2007) or anti-phase (Egidi et al. 2006; Meftah et al. 2016) with the solar activity but some authors reported no obvious variations (Kuhn et al. 2012) (Fig. 1). The longest time series recorded in space, still in progress, was recorded with the Helioseismic and Magnetic Imager (HMI) onboard the Solar Dynamics Observatory (SDO). SDO was launched on February 2010 just after the beginning of Cycle 24 and the series of HMI measurements now covers almost the whole cycle. These measurements are therefore a major asset to explain and validate those carried out in the past, including explaining reported inconsistencies.

\section{HMI DATA AND PROCESSING}

Angular variations of the solar shape and their evolution in time are obtained from roll calibrations performed on SDO/HMI twice a year since its launch. This calibration mode consists of rolling the spacecraft around an axis close to HMI line-of-sight and taking images at constant angular positions during rotation $\left(11.25^{\circ}\right.$ since October 2010). This procedure will later allow removing effects on the solar shape of both mispointing and optical distortion when processing image sequences. Full images of the Sun are recorded in linear polarization at narrow wavelength band $(76 \mathrm{~m} \AA)$ in the solar continuum near the Fe I absorption line at $617.3 \mathrm{~nm}$. They have an angular resolution of 1 arc-second. Their size and angular sampling are $4096 \times 4096$ pixels and 0.5 arc-second, respectively. A complete roll calibration takes about 7 hours while the instrument is subjected to various thermal stresses on its orbit. The method used to process Solar Diameter Imager and Surface Mapper (SODISM) data of the PICARD distortion mode (Irbah et al. 2014) similar to SDO calibration roll, is then applied for HMI image sequences. The processing method is well detailed in Irbah et al. (2014) and the main steps are recalled. The first step is to extract the solar shapes from images of the sequence. The position of the inflection point of the Limb Darkening Function (LDF) for all azimuth angles is calculated for this purpose. LDF angular sampling of SDO images is good enough to detect photometric contaminations due to active regions (sunspots, faculae ...). Our used processing method based on wavelet transform preserves LDF inflection point positions defining the solar shape since bad or hard LDF filtering modifies its slope leading to inflection point shifts (Irbah et al. 1999). Nevertheless, active regions can still affect the mean solar shape computed from all ones of the sequence previously re-phased in azimuth in order to remove effects of both mispointing and optical distortion during averaging. These contaminations appear when solar active regions are localized very close to LDF inflection points. They are, however, easily detectable thanks to the roll procedure properties and then removed during processing. Indeed, rolling the spacecraft causes the solar image to rotate on the HMI CCD camera. All CCD defaults remain on fixed positions in the frame while solar features move with the spacecraft rotation. The roll sequence recorded on July 22, 2015 is taken to illustrate the main steps of the processing method. Azimuthal 


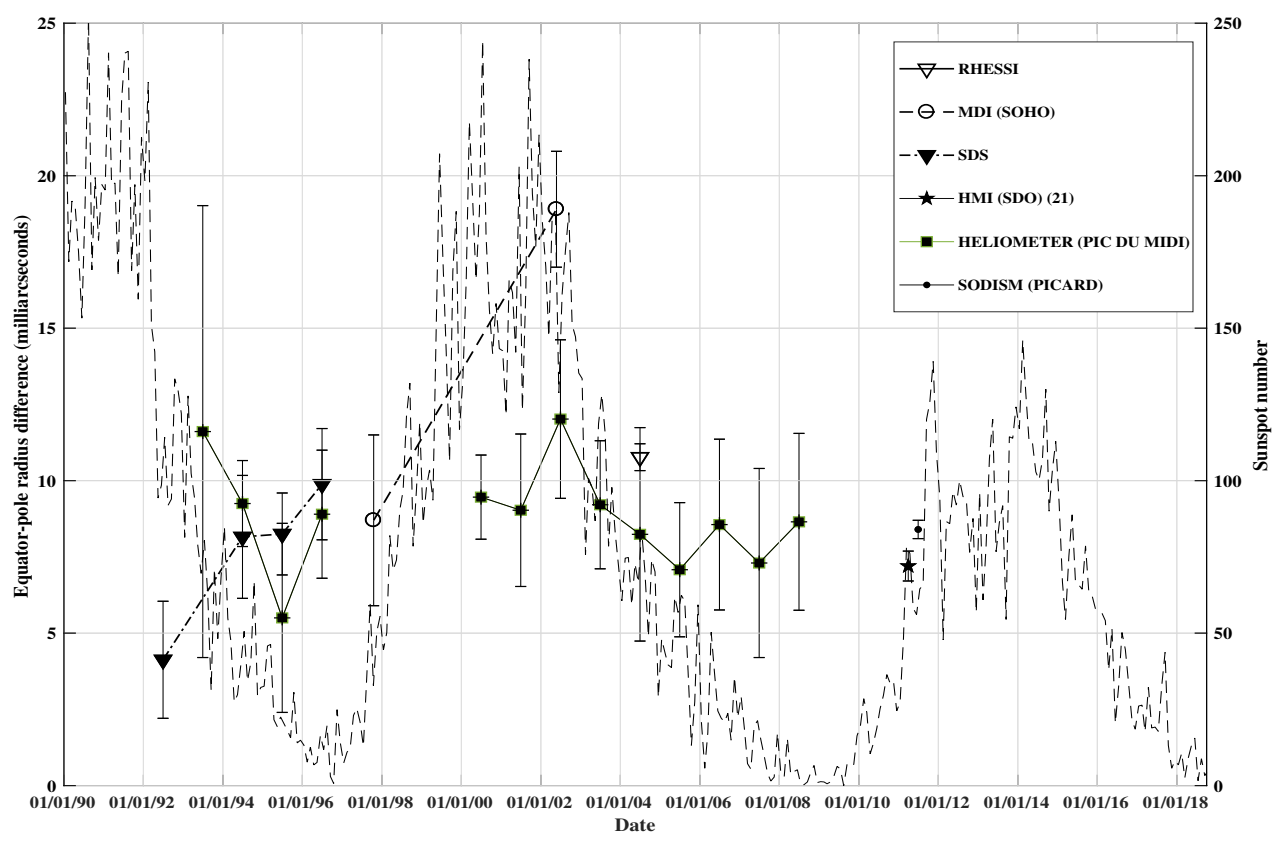

Figure 1. Equator-pole radius difference measured with the Heliometer at the Pic du Midi (France), with SDS on balloon and from Space (RHESSI, MDI, HMI, SODISM) along with sunspot number (SILSO data 2018). The HMI value is the average over the period 2010-2012 of previous measurements since no variations with solar activity were reported (Kuhn et al. 2012). SDS measurements exhibit anti-phase variations in the descending phase of Cycle 22. Measurements made with the Heliometer during Cycle 22 have necessarily important error bars since obtained from ground but they still properly overlap SDS ones. Nevertheless their amplitudes prevent to identify a conclusive phase variation with activity. Space and ground measurements made during Cycle 23 are in phase with solar activity. Note that the first Heliometer measurement of Cycle 23 corresponding to solar activity maximum seems underestimated. This value corresponds to the resumption of measurements with the instrument after an interruption of 2 years (1997-1999).

radius variations extracted from all images of the sequence are shown in Figure 2(a). They are coded in a false color frame where each line is the azimuthal solar radius obtained from one image. Active regions on the extreme solar limb affect the radius at several azimuthal angles. Some effects are shown within the rectangle in Figure 2(a). These effects move in an oblique direction as the spacecraft rotates. Vertical features are also presents but have clearly optical signatures since they remain fixed during roll operations. Figure 2(b) shows all azimuthal solar radius in the same reference frame i.e. the West Equator is the origin of azimuthal angles for all lines. We notice that the solar active regions surrounded by a rectangle in Figure 2(a), are now spread out along the same vertical direction since they are quasi fixed on the Sun surface. The average radius of each line of the frame varies over time during the roll calibration (black curve in Fig. 2(c)). It is then corrected with an iterative process to have the mean radius computed over the entire sequence (red curve in Fig. 2(c)). Finally, the solar shape free of both optical distortion and mispointing effects, is obtained taking the radius mean value for each azimuthal angle (Fig. 2(d)). The angular sampling is $0.2^{\circ}$. A part of the solar shape affected by active regions on the Sun surface is shown in the region surrounded by a rectangle. Figure 2(e) plots the solar shape where solar active regions were removed and a wavelet transform filtering was applied to reduce the noise. The solar shape is then fitted using low-order Legendre polynomials (red curve in Fig. 2(e)). This polynomial fit, up to the fourth order, allows to estimate the quadrupole $c_{2}$ and the hexadecapole $c_{4}$ distortion coefficients. The solar oblateness is then given by $\Delta R=-\frac{3}{2} c_{2}-\frac{5}{8} c_{4}$ where $\Delta R$ is the pole-equator radius difference or $\frac{\Delta R}{R}=-\frac{3}{2} C_{2}-\frac{5}{8} C_{4}$ in case of dimensionless $C_{2}$ and $C_{4} ; R$ is the mean solar radius.

\section{RESULTS AND DISCUSSION}

Sixteen roll sequences, recorded between October 2010 and July 2018, were analyzed according to the processing method presented in Section 2. The resulting average solar oblateness over the period 2010 to 2018 is $8.8 \pm 0.8$ mas 
(a)

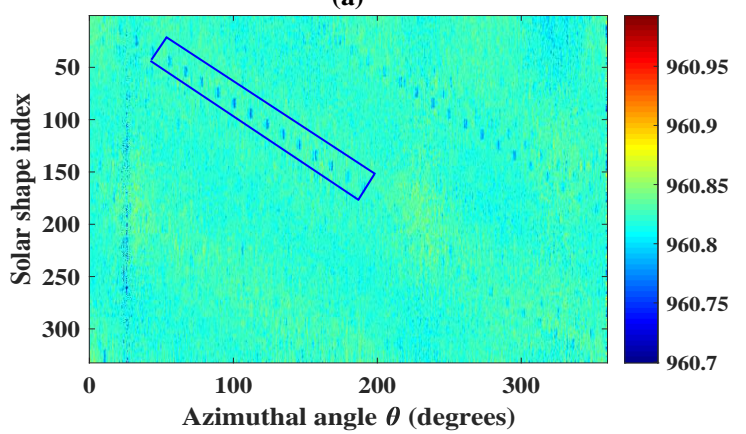

(b)

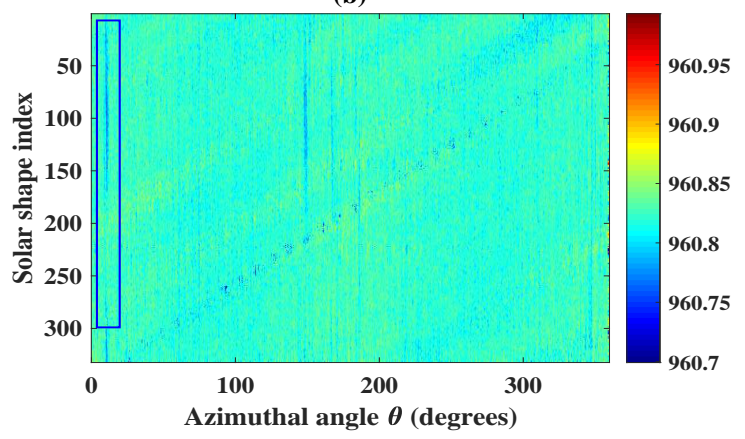

(c)

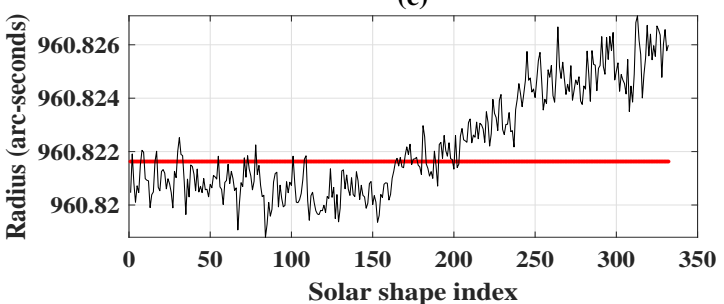

(d)

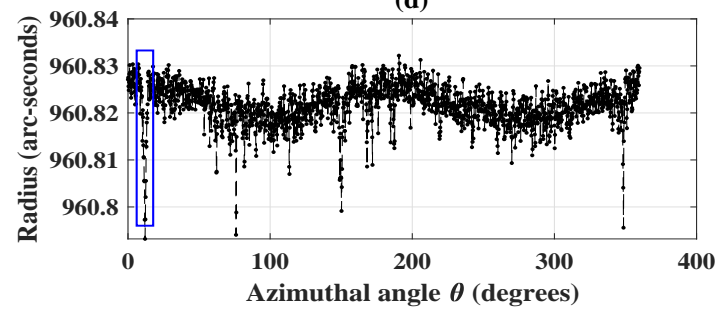

(e)

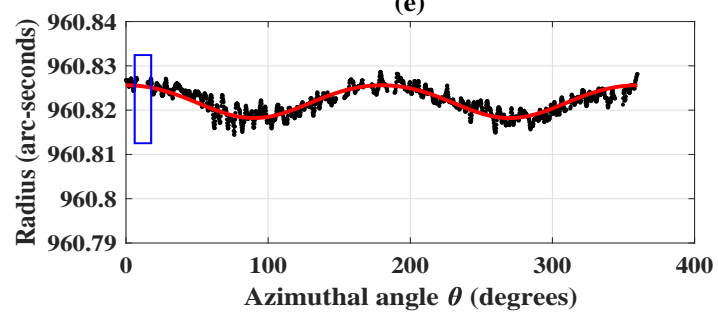

Figure 2. The roll sequence recorded on July 22, 2015 is used to illustrate the processing method. (a) Azimuthal variations of solar radius obtained from images recorded during the roll. Each frame line coded in false colors corresponds to a solar shape calculated from one image. They are 332 images in this sequence and the number of angular samples is 1800 . Some active regions on the Sun (sunspots, faculae ...) as shown by their signature in the rectangle, existed that day and affect the solar shape. (b) Solar shapes are shifted to have all the western equator of the Sun at the zero azimuthal angle. Signatures of active regions affecting the shape are then spread out along frame columns while they are on oblique directions for CCD defaults. (c) A slight time drift of solar radius calculated from each shape (black line) is observed during the roll. It is corrected by mean of an iterative process (red dashed line). (d) All shapes are averaged to compute the mean one where effects of active regions are clearly seen. (e) Active region effects on the solar shape are removed and it is then filtered to reduce the noise. The solar shape is fitted using low-order Legendre polynomials (red curve) to estimate $c_{2}$ and $c_{4}$ distortion coefficients and, then, the oblateness.

corresponding to a radius difference of $6.4 \pm 0.6 \mathrm{~km}$, which is in good agreement with measurements made over the past two decades (Fivian et al. 2008; Irbah et al. 2014; Egidi et al. 2006; Rozelot et al. 2009). They are also consistent with values predicted by helioseismology-based models (Armstrong \& Kuhn 1999; Mecheri et al. 2004). This average value is, however, 1.6 mas higher than that reported by Kuhn et al. (2012) over the period 2010-2012. In addition to the coherent values obtained for oblateness, variations in time of the solar shape are of particular importance in view of the scientific controversies of historical measurements. Results obtained show not only that the solar oblateness exhibits variations, but that they are in anti-phase with the sunspot number taken as proxy for activity of the Sun (Fig. 3(a)). This trend was suspected during the analysis of image sequences taken during the rising phase of solar cycle 24 (Meftah et al. 2016) and it is confirmed by pursuing it on almost the whole cycle. Kuhn et al. (2012), however, reported no change for the period 2010-2012 using the same SDO data. We calculated solar oblateness from the $c_{2}$ and $c_{4}$ values of their paper and found a similar temporal trend as we did (see Fig. 4). It should be noted that the roll calibration of January 24, 2018 gives a value of the solar shape that seems underestimated. It is out of the trend that is emerging, questioning to take it into account or not. The quadrupole $C_{2}$ exhibits variations clearly in phase with solar activity (Fig. 3(b)). Indeed, a linear regression performed between $C_{2}$ values and sunspot number leads to $R^{2} \approx 60 \%$. We can also mention the yearly variations that seem to appear in the temporal evolution of $C_{2}$, in particular during the descent of the cycle of activity. More investigation is needed to find out if these variations are of solar origin or related to orbital correction residues. Concerning the hexadecapole $C_{4}$, we notice that it has anti-symmetric variations relatively to the time of solar activity maximum (Fig. 3(c)). Indeed, $C_{4}$ exhibits variations during both the rising phase 

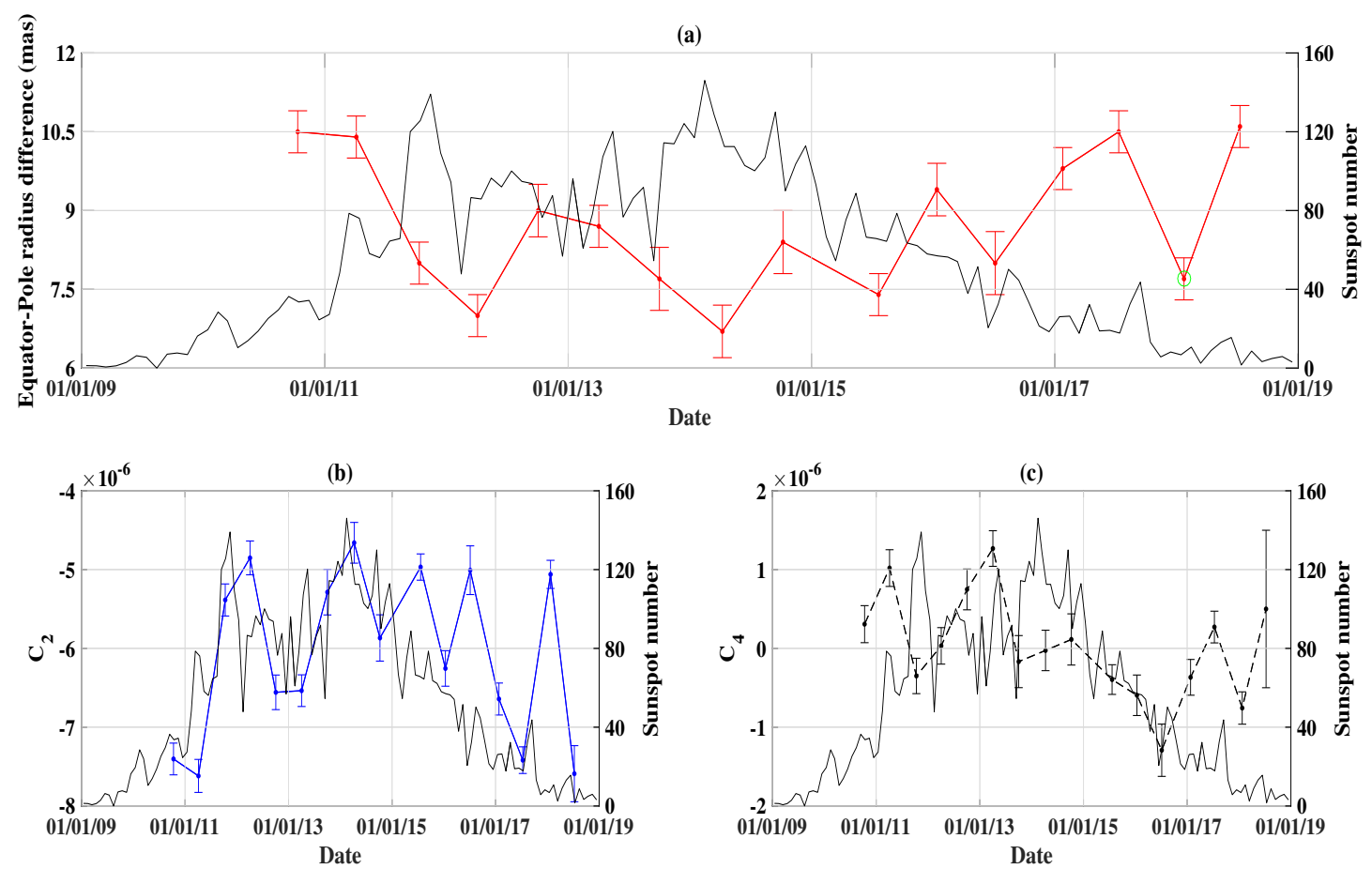

Figure 3. Fit model parameters of equator-pole radius difference obtained from HMI roll calibration images recorded during Cycle 24. (a) Time variations of solar oblateness (red plot) are clearly in anti-phase with the sunspot number (black line) taken as activity proxy. The value surrounded by green is thought to be underestimated due to poor determination and/or sudden solar events. The quadrupole variations $C_{2}$ (blue dots) appear in phase with solar activity (b) whereas the hexadecapole $C_{4}$ (black dots) has anti-symmetric variations relatively to the time of solar activity maximum where they are very small (c).

of Cycle 24 as already reported by Kuhn et al. (2012) for the period 2010-2012 and in the descent of the cycle but of opposite sign. This is clearly seen in Fig. 5 where we fitted $C_{2}$ and $C_{4}$ by sine functions over most of the cycle length. Rozelot et al. (2009) using $C_{2}$ and $C_{4}$ measurements of Emilio et al. (2007), interpreted these temporal variations of the oblateness by a change of the $C_{4}$ values that are insignificant when activity is important, but predominant when activity is low. They suggested that combining both should result in a variation of oblateness in phase with the solar cycle. We observe the same behavior of $C_{2}$ than Emilio et al. (2007) but in anti-phase. For $C_{4}$ we have low values at the extrema (minimum and maximum) of activity and the variations developing with changing activity (rise and fall). We indeed have a series of measurements that allow to understand the variation of $C_{4}$ whereas it is difficult to evidence a behavior with Emilio et al. (2007) since there are only 2 measurements (1997 and 2001), of which one has a large error $(0.3+/-2.5)$ however consistent with our measurements at high activity. We can see in Fig. 3(c) that $C_{4}$ can vary rapidly in a short period of time, which could explain the difference with Emilio et al. (2007) measurement obtained in low solar activity (1997). We can conclude from our results that the solar shape is conditioned by both $C_{2}$ or $C_{4}$ where the first one evolves in phase with solar activity and the second $\left(C_{4}\right)$ varies in phase quadrature with respect to the other. It is worth also to compare their action on the solar shape with that of $a_{2}$ and $a_{4}$, even asphericity coefficients deduced from helioseismology. A recent investigation using both MDI/SOHO and HMI/SDO helioseismic data over 21 years revealed that during solar activity minimum the asphericity of the Sun is dominated by $a_{2}$ and $a_{4}$ splitting coefficients while during solar activity maximum the $a_{2}$ coefficient, which primarily describes the solar oblateness, decreases considerably (Kosovichev \& Rozelot 2018). This result indicates an anti-phase variation of the oblateness with solar activity during Cycle 24 which is in agreement with our results.

Linear regressions are performed on oblateness versus sunspot number considering all measurements performed during Cycle 24 (Fig. 6(a)). The negative slope confirms the anti-phase of oblateness variations with the solar cycle. The linear regression $R^{2}$ is $61 \%$ when all HMI measurements are taken into account and it increases up to $77 \%$ (Fig. 6(c)) when the poor estimated value of January 24, 2018 is removed (value circled in green). The same approach is used for 


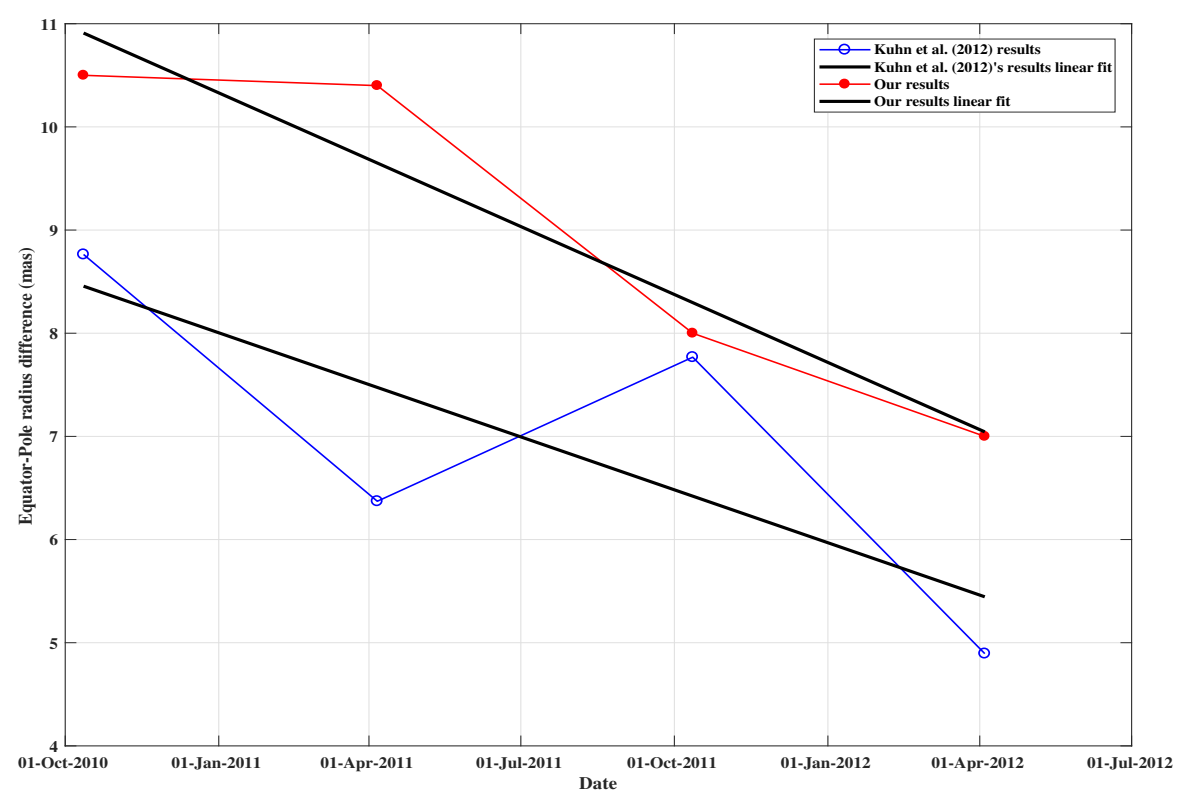

Figure 4. The same temporal trend of solar oblateness exists in Kuhn et al. (2012)'s results similar to ours.

all oblateness measurements made during Cycle 23 with the Heliometer (Damiani et al. 2011), RHESSI (Fivian et al. 2008) and SOHO/MDI (Emilio et al. 2007). The linear regression shows a positive slope with $R^{2}=60 \%$, reflecting oblateness variations in phase with solar activity during Cycle 23 (Fig. 6(b)). The first measurement made in 2000 with the Heliometer seems, however, underestimated. This corresponds to the resumption of measurements after a $2 \mathrm{yr}$ interruption. The linear regression $R^{2}$ increases up to $79 \%$ when this value is removed (Fig. 6(d)). Oblateness measurements performed on balloons with the Solar Disk Sextant (SDS) in the descent of Cycle 22 are in anti-phase with the activity confirming the temporal oscillation of this solar parameter. Taken from ground, measurements with
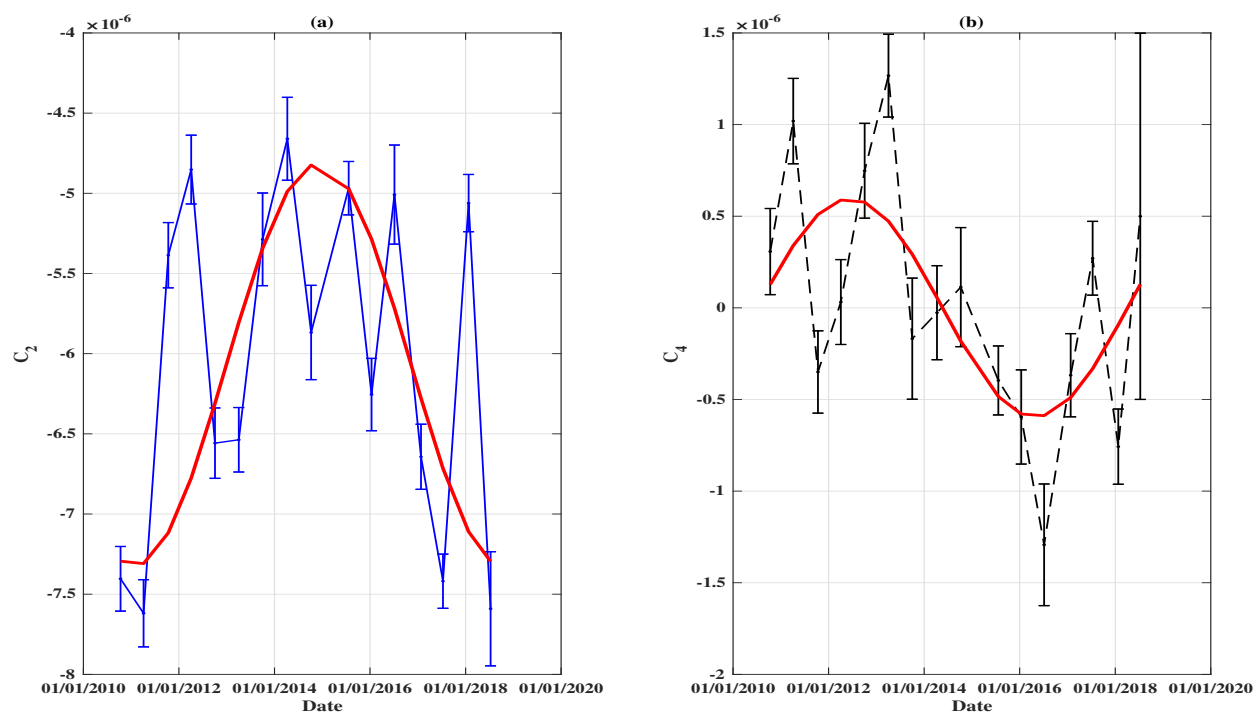

Figure 5. $C_{2}$ (a) and $C_{4}$ (b) temporal trends fitted with sine functions (red full lines) showing how they evolve during solar activity. 

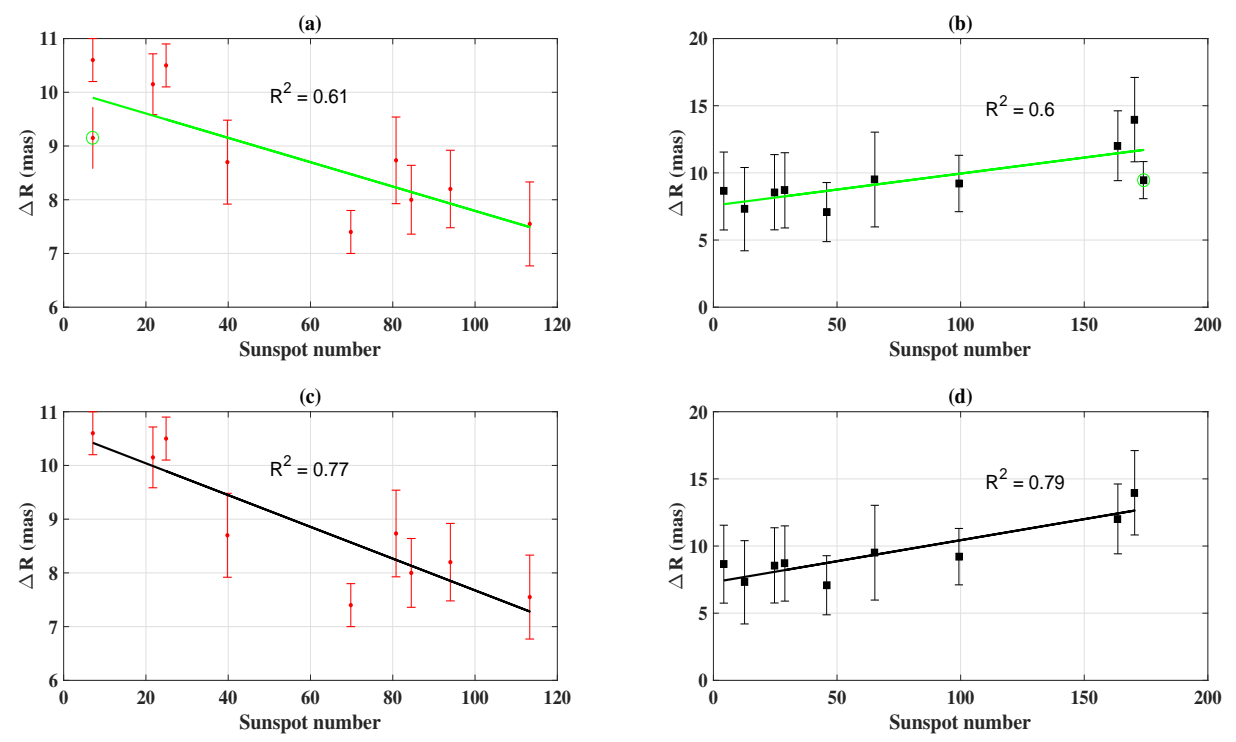

Figure 6. (a) All measurements of equator-pole radius difference made during Cycle 24 are plotted versus sunspot number taken as proxy for solar activity. The linear regression performed on these 2 solar parameters is good giving $R^{2}=61 \%$ (green line). (c) The linear regression is better with $R^{2}=77 \%$ (black line) when the perturbed HMI measurement shown in Fig. 3(a) by a dot surrounded with a green circle is not taken into account. The negative slope shows, as expected, that oblateness variations are in anti-phase with solar activity. (b) Same plot as in (a) when all measurements recorded during Cycle 23 are considered. The linear regression shows a positive slope with $R^{2}=60 \%$ expressing that oblateness variations are in phase with solar activity during Cycle 23 (green line). (d) A better linear regression is obtained with $R^{2}=79 \%$ (black line) when the supposed underestimated value recorded with the Heliometer in 2000 is not taken into account.

the Heliometer made during the same period have naturally a limited precision and larger error bars, but properly overlap those of SDS (Fig. 1), bringing more confidence in their value. Both SDS and the Heliometer had the merit to allow advance in the knowledge of the temporal variability at a time period when very few data were available. It is also worth noting Poor's analysis (1905) of earlier oblateness measurements made during Cycles 11 and 13, as well as Ambronn and Schur ones (1905) made during Cycles 11 and 12 (see Fig. 1 in Damiani et al. (2011)). Despite the limited precision of these historical measurements, variations although very large, are clearly in phase with solar activity of Cycles 11 and 13 whereas, during Cycle 12, they are very similar to HMI variations observed around the maximum of solar activity, that is to say in anti-phase. Accordingly, it is possible that the Sun initiates a physical process that results in a pulsation with a period of twice the 11yr solar cycle. It has maximum swelling during odd cycles and vice versa for even ones, i.e. the solar shape oscillates like the magnetic field having extreme values during its polarity inversion. It is therefore the time of the measurements, with respect to the temporal oscillation of solar oblateness, that largely explains the controversy surrounding past measurements reported in the literature. Why oblateness oscillates with the 22yr magnetic cycle of the Sun is another challenge to model, which could probably be addressed by precise diagnostics of surface layers structure and dynamics. The complex structure of the leptocline, as defined in Reiter et al. (2015), is certainly a key to understand the described mechanism.

\section{REFERENCES}

Antia, H. M., Chitre, S. M. \& Thompson, M. J.: 2000,

Astron. Astrophys, 360, 335

Antia, H. M., Chitre, S. M. \& Gough, D. O.: 2008, Astron.

Astrophys, 477, 2, 657

Armstrong, J. \& Kuhn, J. R.: 1999, Apj, 525, 1, 533

Chapman, R.: 2008, Science, 322, 535
Damiani, C., Rozelot, J.P., Lefebvre, S., Kilcik, A. \&

Kosovichev, A.G.: 2011, JASTP, Issue 2-3, 241

Dicke, R. H. \& Goldenberg, H. M.: 1974, ApJ Supp. Series, $241,24,131$

Dicke, R. H., Kuhn, J. R. \& Libbrecht, K. G.: 1987, ApJ, 318, 451 
Egidi, R., Caccin, B., Sofia, S. et al.: 2006, Sol. Phys., 235, 410

Emilio, M., Bush, R.I., Kuhn, J., Sherrer, P.: 2007, ApJ, 660, L161

Fivian, M., Hudson, H., Lin, R., Zahid, H.: 2008, Science, 322,560

Goode, P. R. \& Thompson, M. J.: 1992, ApJ, 395, 307

Hill, H.A. \& Stebbins, R.T.: 1975, ApJ, 200, 471

Irbah, A., Meftah, M., Hauchecorne, A., Djafer, D. et al.: 2014, ApJ, 785, 89

Irbah, A.; Bouzaria, M., Lakhal, L., Moussaoui, R. et al.: 1999, Sol. Phys., 185, 2, 255

Kuhn, J.R., Bush, R., Emilio, E., Scholl, I.F.: 2012, Science, 337, 1638

Kosovichev, A.G. \& Rozelot, J.P.: 2018, JASTP, 176, 21

Lefebvre, S., Rozelot, J. P. \& Kosovichev A. G.: 2007, ASR, 40, 7, 1000

Le Verrier, U. J.: 1859, Ann. Obs. Paris, 5, 1
Mecheri, R., Abdelatif, T., Irbah, A., Provost, J., Berthomieu, G.: 2004, Sol. Phys., 222, 2, 191

Newcomb, S.: 1865, Constants of astronomy, US GPO, Washington, DC, 111

Newcomb, S.: 1895, The Elements of the Four Inner Planets, Bulletin Astronomique, Serie I, 13, 26

Meftah, M., Hauchecorne, A., Bush, R.I. \& Irbah, A.: 2016, ASR, 58, 7, 1425

Paterno, L., Sofia, S. \& Di Mauro, M. P.: 1996, Astron. Astrophys, 314, 940

Reiter, J., Rhodes, E. J., Jr., Kosovichev, A. G., Schou, J. et al.: 2015, ApJ, 803, 2, 92

Rozelot, J.P. \& Damiani, C.: 2011, Eur. Phys.J. H, 36, 407

Rozelot, J.P., Damiani, C. \& Pireaux S.: 2009, ApJ, 703, 1791

SILSO data/image, Royal Observatory of Belgium, Brussels

Sturrok, P. A. \& Gilvarry J. J.: 1967, Nature, 216, 1280 